Check for updates

Saw Swee Hock School of Public Health, National University of Singapore, Singapore

2 Ministry of National Guard Health Affairs, Saudi Arabia

3 King Saud bin Abdulaziz University for Health Sciences, Riyadh, Saudi Arabia

4 Last Mile Health, Boston. MA, USA

5 Department of Medical Affairs and Planning, Taipei Veterans General Hospital, Taiwan

6 National Yang-Ming University Institute of Hospital and Health Care Administration, Taiwan

7 University of Hong Kong, Hong Kong

8 The BMJ, London, UK

9 Bioinformatics Institute, Agency for Science, Technology and Research, Singapore

Correspondence to M-K Looi mlooi@bmj.com Cite this as: $B M J 2021 ; 372: n 486$ http://dx.doi.org/10.1136/bmi.n486 Published: 11 March 2021

\title{
How the lessons of previous epidemics helped successful countries fight covid-19
}

Covid-19 caught the West by surprise, but the legacy of Ebola, MERS, and SARS meant that several Asian and African nations had systems in place to mitigate the severity of the pandemic. Experts explain what Hong Kong, Liberia, Saudi Arabia, Singapore, and Taiwan learnt and how they have made a difference.

Alvin Qijia Chua, ${ }^{1}$ Bandar Al Knawy, ${ }^{2,3}$ Ben Grant, ${ }^{4}$ Helena Legido-Quigley, ${ }^{1}$ Wui-Chiang Lee, ${ }^{5,6}$ Gabriel M Leung, ${ }^{7}$ Mun-Keat Looi, ${ }^{8}$ Sebastian Maurer-Stroh ${ }^{9}$

\section{Hong Kong-Scars of past epidemics}

\section{Gabriel M Leung, University of Hong Kong}

East Asia's response to covid-19 seems to have been exceptional so far. This is-at least partly-a result of the many lessons learnt and innovations derived from the region's decades-long experience with outbreaks of novel, directly transmissible respiratory pathogens. These include the 1957 “Asian” and 1968 "Hong Kong” influenza pandemics, the first occasion of $\mathrm{A}\left(\mathrm{H}_{5} \mathrm{~N}_{1}\right)$ influenza jumping the species barrier from birds into humans in 1997, and the $\mathrm{A}\left(\mathrm{H}_{7} \mathrm{~N} 9\right)$ in 2013 around the Yangtze River Delta. The 2002-03 SARS epidemic left the most indelible mark. ${ }^{1}$

The sociological imprints left by these outbreaks have cumulatively boosted people's adherence to, and even demand for, the public health and social measures that have proved so critical to controlling covid-19. This is all the more remarkable in Hong Kong, given the large deficit of trust in government following massive social unrest in 2019. ${ }^{2}$

After SARS, the Hong Kong government adopted a clearly defined, tiered command structure to prepare for and respond to future outbreaks, and consolidated all health protection functions under a new centralised agency. ${ }^{3}$ In parallel, massive investment has gone into research preparedness, with dialogue between the scientific and policy making communities in real time. Ever more stringent border restrictions have been adopted, ${ }^{4}$ and quarantine arrangements for both imported and local patients have improved to the extent that the Wall Street Journal found them to be the best in the world. 5

\section{Liberia-Resilient community health systems}

\section{Ben Grant, Last Mile Health}

Strong community based health systems respond quickly and effectively to epidemics. In 2016, Liberia's ministry of health designed and launched the National Community Health Assistant Program (NCHA), which to date has recruited, trained, and deployed more than 3500 community health workers. These workers are linked to 316 primary care clinics and serve more than 800 ooo people in rural and remote communities.

Until 2016, community health services were fragmented, with parallel programmes and little standardisation across the country. The Ebola epidemic of 2014 highlighted significant gaps in the primary healthcare system and brought inequities into sharp focus. The new, harmonised, NCHA programme helped bridge the gaps, ensuring that all communities more than $5 \mathrm{~km}$ from a health facility had access to essential lifesaving services, and that no Liberian was out of reach.

Community health workers have laid the foundation for a more resilient primary healthcare system, which includes surveillance, diagnostics, and therapeutic services. During the Ebola outbreak, health workers' relationships with their communities helped address mistrust and stigma surrounding the disease. Conversely, late engagement by community health workers hindered efforts to manage the outbreak. ${ }^{67}$ Outsiders sent to affected communities were rejected and their efforts were counterproductive.

As Liberia responds to its second major disease outbreak in five years, community health workers are vital to prevent, detect, and respond to covid-19. When SARS-CoV-2 was detected, health workers were quickly trained, equipped, and deployed to respond at the community level, while being closely linked with health facilities. Training included identifying suspected cases, surveillance, contact tracing, referral protocols, and supporting patients in home isolation. Based on lessons of the no-touch policy used by community health workers during the Ebola outbreak, protocols were adjusted to keep workers safe as they provided lifesaving screening and treatment for malaria, diarrhoea, pneumonia, and malnutrition and supported access to family planning, immunisations, prenatal care, and facility based delivery.

Prioritising and investing in community health workers enables a quick response to epidemic events, especially in remote and rural areas. For the pandemic response in Liberia, community health workers will continue to be a powerful mechanism for addressing misinformation and vaccine hesitancy, through training and relationship building in their communities. These workers are vital for emergency and everyday service delivery that ensures quality and equitable health services. 


\section{Saudi Arabia-Drive-through screening and vaccine development}

\section{Bandar Al Knawy, King Saud bin Abdulaziz University for Health Sciences}

The outbreak of MERS in 2012 resulted in hundreds of deaths and spread to 27 countries, causing worldwide concern of a potential pandemic. Its emergence in the Kingdom of Saudi Arabia triggered transformational changes in the country's healthcare system. This proved critical in launching a prompt response to covid-19.

The MERS outbreak highlighted that delays in identifying cases and improper triage in emergency departments were the main risks for nosocomial outbreaks. ${ }^{8}$ In response to a MERS outbreak in our tertiary hospital in 2015, we introduced an innovated triaging process at the emergency department; whereby patients were directed into two physically independent pathways according to the presence or absence of respiratory symptoms. This was determined using a screening system that comprised drive-through booths where patients undertook a 60 second questionnaire. The system showed $100 \%$ sensitivity in detecting patients with MERS (later confirmed by polymerase chain reaction testing) ${ }^{9}$ and helped reduce transmission of MERS in the hospital.

The health ministry launched a multi-dimensional research programme for MERS that resulted in important clinical trials in therapeutics and vaccine development. In collaboration with Oxford University, a MERS vaccine based on a chimpanzee adenovirus vector (ChAdOx1) was developed and tested in healthy humans in phase I trials in Saudi Arabia and the UK, and was found to be well tolerated, with no serious adverse events. ${ }^{10} \mathrm{~A}$ phase II trial is planned collaboratively with Oxford University, funded by the Coalition for Epidemic Preparedness Innovations. The trials supported the swift development of a ChAdOx1 based vaccine for covid-19, while also harnessing the genetic similarities between MERS-CoV and SARS-CoV-2.

\section{Singapore-A holistic approach}

\section{Alvin Qijia Chua, Helena Legido-Quigley, Saw Swee Hock School of Public Health}

\section{Sebastian Maurer-Stroh, Bioinformatics Institute, Agency for Science, Technology and Research}

In response to the 2003 SARS outbreak, the Singapore government built a multi-ministry taskforce to coordinate actions, centralise efforts, and disseminate information about the epidemic. Similar taskforces were set up for the $2009 \mathrm{H} 1 \mathrm{~N} 1$ pandemic, the 2016 Zika outbreak, and most recently for covid-19.

After the 2003 epidemic a Disease Outbreak Response System Condition framework was drafted. ${ }^{11} \mathrm{~A}$ five-colour alert system indicates the current outbreak situation and provides guidance to the public on actions to prevent and reduce the impact of infections.

This is paired with a timely and accurate surveillance system. During SARS, the ministry of health introduced surveillance measures to integrate epidemiological data and to identify emerging virulent strains more quickly. This initiative included community, laboratory, veterinary, hospital, and external surveillance. An Infectious Disease Alert and Clinical Database system was also set up to pool clinical, laboratory, and contact tracing information. ${ }^{12}$ In 2006 this was matched with a live online portal that allowed physicians to submit timely notification of infectious diseases and to access real time information on local and global infectious disease events.
In primary care, physicians have been trained and prepared for infectious disease outbreaks, enabling them to identify and triage high risk patients quickly. Treatments, including medication and vaccinations, are subsidised by the government for patients presenting with respiratory symptoms. ${ }^{2}$ During the H1N1 pandemic, many private primary care clinics were activated to facilitate these treatments under the Pandemic Preparedness Clinic scheme. In 2015 the scheme was updated and the clinics became Public Health Preparedness Clinics (PHCPs) which treated other health problems, such as respiratory issues arising from haze air pollution. These PHPCs were reactivated during the covid-19 pandemic. Currently, patients with respiratory illness pay up to $S \$ 10$ (£5.30; US $\$ 7.50$; $€ 6.25)$ for their treatment, and the rest is covered by government subsidy.

Containment measures, such as early detection and isolation of all cases and quarantine of close contacts, were implemented in 2003 to prevent community transmission of SARS. ${ }^{13}$ These were implemented again in the $\mathrm{H}_{1} \mathrm{~N}_{1}$ and covid-19 pandemics. ${ }^{14} 15$

Over the years, technology advances have strengthened containment further. SafeEntry, a national digital location check-in system ensures that individuals visiting public spaces, including workplaces and social venues, have checked in to the venue using a $Q R$ code. The complementary TraceTogether application or token uses Bluetooth signals exchanged between mobile phones to identify close contacts of a positive case.

Advances in genome sequencing have also helped. In 2003 it took two months for the genome of the SARS index case to be released on public databases, but it took less than two weeks for the first local SARS-CoV-2 genome to made available in 2020. ${ }^{16}$ One year into the covid-19 pandemic, genomes are often released less than a week after sample collection. The National Public Health Laboratory conducts whole genome sequencing and phylogenetic analysis on all cases meeting defined criteria as part of routine laboratory surveillance. This is complemented by other local institutions to ensure the system has surge capacity.

Quarantine facilities during SARS included holiday resorts, and this has been expanded during covid-19 to include Singapore Armed Forces camps, the Singapore EXPO. and Changi Exhibition Centres, and hotels. Legislation passed in April 2020 allows the government to requisition any land, undertaking, or other resource for the purpose of control order. It also provides a legal basis to enforce social distancing measures, such as limiting mass gatherings and holding individuals and business owners accountable for violation with fines, jail, and other penalties.

Following SARS, simulation exercises were organised at various public hospitals to rehearse outbreak scenarios and to inform plans to manage outbreaks. ${ }^{17}$ National stockpiles of personal protective equipment and essential medicines are maintained for up to six months. ${ }^{18} \mathrm{~A}$ lack of adequate treatment facilities during SARS prompted the building of a specialised infectious disease centre to be mobilised during future outbreaks. The National Centre for Infectious Diseases, inaugurated in 2019, has 330 beds with integrated clinical, laboratory, and epidemiological functions. It houses a screening centre, isolation and cohort wards, intensive care units, laboratories, imaging facilities, and operating theatres specifically for infectious disease patients. It also has an inbuilt real time location system to track the location of assets and patients, and to aid contact tracing efforts in the event of an outbreak. 


\section{Taiwan-Central coordination}

Wui-Chiang Lee, Department of Medical Affairs and Planning, Taipei Veterans General Hospital, Taiwan; National Yang-Ming University Institute of Hospital and Health Care Administration, Taiwan

After the painful experience of SARS in 2003, the Taiwanese government realised that its Centers for Disease Control (CDC) and the Communicable Disease Control Act (CDCA) had been insufficient to respond to large scale contagious diseases effectively and efficiently. The containment strategies of central and local government had been uncoordinated, and preparedness in some hospitals was inadequate.

In response, the government undertook a fundamental reorganisation of the $\mathrm{CDC}$, recruiting experts in infection control and public health, and establishing a new unit charged with collecting and analysing information on infection. A special team of 30 physicians was put in place to look at emerging diseases.

The government also amended the CDCA, establishing an ad hoc Central Epidemic Command Center (CECC) that would coordinate ministries, public and private organisations, and information and logistics at a national level in the event of a pandemic. The CECC was quickly activated on 20 January 2020 and put on its highest alert level on 27 February. Immediately it took a series of preventive measures, including tight border control, travel restrictions, precision testing (of people with symptoms, signs of infection, travel to a high risk country, or who had contact history with a suspected case), and organised isolation and quarantine at contracted hotels rather than homes to contain infections. The CECC also introduced mandatory mask wearing and social distancing, banned the exportation of personal protective equipment, and boosted domestic mass production of the materials needed to make more. The CECC has also hosted daily press conferences, and gave a briefing on each new case's route of infection and the individual's health status (while protecting patient privacy).

As a result, Taiwan has had only around 600 positive cases of covid-19.

Competing interests: We have read and understood The BMJ policy on declaration of interests and have no relevant interests to declare.

Provenance and peer review: commissioned, not peer reviewed.

1 Shibuya K, Tan CC, Chun AY, Leung GM. The rising role of East Asia in global health security. PLoS Med 2021; [forthcoming].

2 Ni MY, Yao XI, Leung KSM, etal. Depression and post-traumatic stress during major social unrest in Hong Kong: a 10-year prospective cohort study. Lancet 2020;395:273-84. doi: 10.1016/S0140-6736(19)33160-5 pmid: 31928765

3 SARS Expert Committee. 2003. https://www.sars-expertcom.gov.hk/english/reports/reports/reports_fullrpt.html

4 Leung GM, Cowling BJ, Wu JT. From a sprint to a marathon in Hong Kong. N Engl J Med 2020;382:e45. doi: 10.1056/NEJMc2009790 pmid: 32294373

5 Which countries have responded best to Covid-19? Wall Street Journal 2020. https://www.wsj.com/articles/which-countries-have-responded-best-to-covid-19-11609516800

6 Miller NP, Milsom P, Johnson G, etal. Community health workers during the Ebola outbreak in Guinea, Liberia, and Sierra Leone. J Glob Health 2018;8:020601.pmid: 30023054

7 Luckow PW, Kenny A, White E, etal. Implementation research on community health workers' provision of maternal and child health services in rural Liberia. Bull World Health Organ 2017;95:113-20. doi: 10.2471/BLT.16.175513 pmid: 28250511

8 Al Knawy BA, Al-Kadri HMF, Elbarbary M, Arabi Y, Balkhy HH, Clark A. Perceptions of postoutbreak management by management and healthcare workers of a Middle East respiratory syndrome outbreak in a tertiary care hospital: a qualitative study. BMJ Open 2019;9:e017476. doi: 10.1136/bmjopen-2017-017476 pmid: 31061009

9 Alsolamy S, Khan A, Yousef S, Alghusen A, Alnaim A, Alsalamah M. Sensitivity and specificity of a Middle East respiratory syndrome screening tool used in the emergency department. / Nat SCi Med 2020;3:44-7doi: 10.4103/JNSM.JNSM_15_19.
10 Folegatti PM, Bittaye M, Flaxman A, etal. Safety and immunogenicity of a candidate Middle East respiratory syndrome coronavirus viral-vectored vaccine: a dose-escalation, open-label, non-randomised, uncontrolled, phase 1 trial. Lancet Infect Dis 2020;20:816-26. doi: 10.1016/S1473-3099(20)30160-2 pmid: 32325038

11 Lee CE, Satkunanantham K, eds. Singapore's health care system: What 50 years have achieved. World Scientific, 2016doi: 10.1142/9648

12 Lai AY-H, Tan TB. Combating SARS and H1N1: insights and lessons from Singapore's public health control measures. ASEAS 2012;5:74-101.

13 Ooi PL, Lim S, Chew SK. Use of quarantine in the control of SARS in Singapore. Am I Infect Control 2005;33:252-7. doi: 10.1016/j.ajic.2004.08.007 pmid: 15947741

14 Chua AQ Tan MMJ, Verma M, etal. Health system resilience in managing the COVID-19 pandemic: lessons from Singapore. BMJ Glob Health 2020;5:e003317. doi: 10.1136/bmigh-2020-003317 pmid: 32938609

15 Tay J, Ng YF, Cutter JL, James L. Influenza A (H1N1-2009) pandemic in Singapore--public health control measures implemented and lessons learnt. Ann Acad Med Singap 2010;39:313-12.pmid: 20473458

16 Pung R, Chiew CJ, Young BE, etalSingapore 2019 Novel Coronavirus Outbreak Research Team. Investigation of three clusters of COVID-19 in Singapore: implications for surveillance and response measures. Lancet2020;395:1039-46. doi: 10.1016/S0140-6736(20)30528-6 pmid: 32192580

17 Lum LH, Badaruddin H, Salmon S, Cutter J, Lim AY, Fisher D. Pandemic preparedness: nationally-led simulation to test hospital systems. Ann Acad Med Singap 2016;45:332-7.pmid: 27683737

18 Goh KT, Cutter J, Heng BH, etal. Epidemiology and control of SARS in Singapore. Ann Acad Med Singap 2006;35:301-16.pmid: 16829997

This article is made freely available for use in accordance with BMJ's website terms and conditions for the duration of the covid-19 pandemic or until otherwise determined by BMJ. You may use, download and print the article for any lawful, non-commercial purpose (including text and data mining) provided that all copyright notices and trade marks are retained. 